Cell Research (1999), 9, 209-216

\title{
Cloning and analysing of 5' flanking region of Xenopus organizer gene noggin
}

\author{
TAO Qin Hua, Jing YANG, Wen Yan MEI, Xin \\ GENG, XIAO YAN DING* \\ Shanghai Institute of Cell Biology, CAS, Shanghai 200031, \\ China
}

\begin{abstract}
Xenopus organizer specific gene Noggin possesses nearly all the characterestic properties of the action of organizer to specify the embryonic body axis. To analyze how the maternal inherited factors control its expression pattern, we cloned the 5 ' regulatory region of noggin gene. The $1.5 \mathrm{~kb}$ upstream sequense could direct reporter gene to express in vivo and data from deletion analysis indicated that a 229 base pair fragmet is essential for activating noggin expression. We further demonstrated that the response elements within this regulatory region were indeed under the control of growth factor activin and Wnt signaling pathway components.
\end{abstract}

Key words: Xenopus, organizer specific gene, noggin, regulatory region

\section{INTRODUCTION}

The dorsal blastopore lip of Xenopus early gastrulae plays a key role in specifying the body axis. Transplanting a small piece of dorsal lip into the ventral side of another embryo causes the formation of a secondary axis, resulting in a twinned embryo and thus, the dorsal lip of blastopore is named "the organizer"[1]. Due to the functional importance of the organizer, intense efforts have been placed on deciphering its molecular properties and in past decades dozens of organizer genes have been identified(reviewed by Heasman J[2]).

The organizer is progressively induced during blastula stage by maternal inducing signals. It's generally accepted that mesoderm-inducing signals and dorsal determinants

* Corresponding author, e-mail: xyding@sunm.shcnc.ac.cn 
5' regulatany region of noggin gene

are both necessary for the formation of the organizer[2]. The most promising candidates of mesoderm-inducing signals are factors encoded by genes of the members of transforming growth factor-beta (activin, Vg1, BMP-4, nodal related genes etc.) and fibroblast growth factor super families[3],[4]. Increasing evidence also revealed that components of Wnt signaling pathway have been implicated in dorsalization of mesoderm[2]. These two kinds of maternal signals orchestrated in the vegetal dorsal territories of blastula, resulting in the establishment of the organizer. However, how these maternal inherited factors induce the formation of the organizer, in terms of initiating the expression of organizer spesific genes, remains unclear.

NOGGIN, a secreted factor, is expressed in the organizer region during gastrulation and has nearly all characterestic properties of the organzier, such as in patterning body axis, dorsalizing ventral mesodrm and converting prospective ectoderm to neural ectoderm. It can also induce an imcomplete additional body axis when mis-expressed in ventral marginal zone[5]. Therefore, to analyze the regulation of noggin expression will help greatly in understanding the establishment of the organizer. To this aim, we cloned a 1. $5 \mathrm{~kb}$ noggin 5' upstream DNA by screening a partial Xenopus genomic library. Reporter gene assays indicated that this genomic sequence was able to direct reporter gene to express in vivo and respond to activin and Wnt signalings. Furthermore, a $229 \mathrm{bp}$ fragment has been identified to be essential for the basal promoter activity by serial deletion analysis.

\section{MATERIALS AND METHODS}

\section{Isolation of Xenopus noggin genomic sequence}

Xenopus genomic DNA were digested with EcoRI and fractionated by $0.7 \%$ agarose gel, then recovered according to molecular weight ladder $(1-2,5-6 \mathrm{~kb}$, and over than $6 \mathrm{~kb}$. A pair of PCR primers specific to noggin 5' untranslated region (UTR) was used to probe the genomic fragments. Since the positive signal was detected in the $2-3 \mathrm{~kb}$ genomic fragments with the highest abundance (see Fig 1A), a special library containing the EcoRI digested 2-3 kb genomic fragments was constructed in pBluescript II SK- vector for further screening. By using the probe amplified from the 5'UTR of noggin cDNA, one positive clone was screened out from about 200,000 transformants. Sequencing result indicated that the positive insert was 2066 base pair covering 516 base pair 5' UTR of noggin cDNA and $1550 \mathrm{bp} 5$ 'flanking region. The positive clone was therefore named pSK-N2066. PCR primers specific to noggin 5'cDNA are as follows.

foreward: 5'actttctctggttgcatccc 3'; reverse: 5'acctagcgaattggggattc 3 '.

\section{Construction of expression plasmids}

Functional beta-galactosidase gene was cloned from pCH110 (phamarcia) with BamHI/HindIII (blunted) into pSK-N2066 with BamHI/SmaI. This reporter construct was named 2066-LacZ.

1556bp 5' flanking region was amplified from pSK-N2066 by PCR with T7 primer and noggin specific primer 5' gectggctaatggatcctaagtagcc 3' (1545-1570 bp in Fig 1B, introducing a BamHI site by substituting aa with gg bases for cloning convenience), and inserted into pBluescript SKvector with EcoRI/ BamHI sites. The resultant was named pSK-N1556. Serial deletions were obtained by the following processes: pSK-N1556 were double digested with either EcoRI/NdeI, EcoRI/StuI, EcoRI/HindIII or HindIII/StuI, blunted by Klenow and then religated, resulting in 
Tao $\mathrm{QH}$ et al.

serial deletions: pSK-N797, pSK-N657, pSK-N329 and pSK-N1556(-229). The 1556 bp 5' flanking fragment and its serial deletions were then cloned into firefly luciferase based pGL3-basic with $\mathrm{KpnI} / \mathrm{BamHI}$ and $\mathrm{KpnI} / \mathrm{BglII}$ sites, and respectively named as 1556-Luc, 797-Luc, 657-Luc, 329Luc and 1556(-229)-Luc.

\section{Preparation of synthetic RNA}

The plasmid psp64T-activin bb (a gift from Harland RM) was linearized with XbaI and transcripted by SP6 RNA polymerase, pCDNA1.1-ArmdNXTcf-3 (a gift from Roose J), which encodes the fusion protein containing the activation domain (694-844 aa) of Drosophila Armadillo and N-terminus (1-31 aa) deleted Tcf-3 of Xenopus, and has the constitutive dorsalizing activitiy, was linearized with XbaI and transcripted by T7 RNA polymerase. Both reactions were carried out with cap analog/GTP ratio 5:1.

\section{Beta-galactosidase staining and luciferase assay}

Albino Xenopus embryos injected with beta-galactosidase reporter gene were fixed at various stages, and stained with X-Gal. The staining procedure was described previously[6].

For dual-luciferase reporter assays, animal caps and embryos were collected and excess medium was removed. Each sample was triplicated. $10 \mu \mathrm{l}$ of $1 \times$ Passive Lysis Buffer per animal cap or per whole embryo was added to homogenize the samples, and $10 \mu \mathrm{l}$ of lysate was used to do luminescence measurement (See details in Dual-Luciferase reporter gene assay system user book, Promega)

\section{Handling and manipulation of embryos}

All embryos were obtained by artificial insemination, de-jellied in $2 \%$ cystein-Cl-( $\mathrm{pH} 7.8$ ), reared in $0.1 \times$ modified Barth saline (MBS) or with additional $1 \%$ Ficoll 400 ( Sigma) for microinjection, and staged according to Nieuwkoop and Faber (1967)[7]. In beta-galactosidase reporter assay, albino embryos were used.

For promoter activity assay, dual-luciferase reporter gene assay system (Promega) was used. Luciferase reporter constructs combining with internal control pRL-SV40 (1:5 in concentration) were injected at 4 to 8 -cell stage in dorsal marginal zone. Injected embryos were harvested at stage 10.5 for luminescence measurement. For animal cap assays, dual-luciferase reporter genes were injected either with or without 1-2 pg mRNA of interests (actvin or ArmdNXTcf-3) at 2-cell stage in animal pole. The animal caps were dissected at stage 8 and reared in $1 \times$ MBS to stage 10.5 for luminescence measurement.

All injected embryos were reared in $0.1 \%$ Ficoll, $0.1 \times$ MBS till stage 7 , then transferred to 0 . $1 \times$ MBS for further incubation.

\section{RESULTS AND DISCUSSION}

\section{Isolation of 5 ' flanking region of Xenopus organizer gene noggin}

A $2066 \mathrm{bp}$ long genomic sequence was obtained from a special fractionated genomic library by a noggin 5'UTR specific probe (see details in Materials and Methods). Sequencing indicated that this genomic sequence covered $516 \mathrm{bp}$ of 5' UTR and $1550 \mathrm{bp}$ of 5 ' flanking region.

The promoter activity of this genomic fragment was examined by injecting reporter construct 2066-LacZ at 2-cell stage in marginal zone of one cell. In reporter construct 2066-LacZ, the functional betagalactosidase gene was inserted immediately downstream to $2066 \mathrm{bp}$ of genomic fragment. X-Gal staining through gastrula to taibud stages em- 
5' regulatany region of noggin gene

A

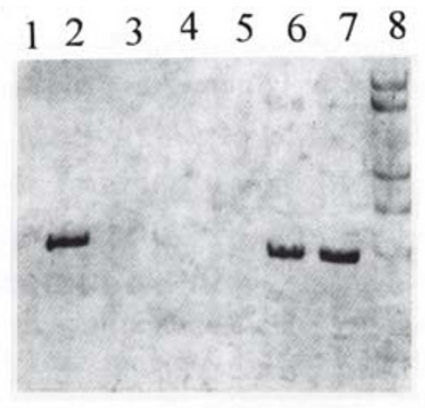

B 1

GAATTCTGCA TTTGGGTGGC ATTTAATATT TGCAAAAAAT AAAAACCAGC EcoRI

ATGGTACTAA AATGCAATTA ACCCCCCATC CCCATAAGAT AAATGTGTCC CCTTGTAAAA AGATATTGAT CACTTCTACA GACAACTTGT ATGGAAATGG GTACATTAAC CATTAACATT CTGGCAGCAT CTGCTTGTTC TAGTGCACAA GGGTTAATTC AGTGTATCAG TGCATGGCAT CACTTTCTCT ATTTTCTGGG TAAAAAAAAT AGCGTTAACC CTTAATGTAC CATACATCTT ATACCTGAAG AAACCCAGGC CATTTACTGC GCCCTTGCCC ACTGTAATGT CAATTCTTAG CAAATAAAAT GTCCCATGTT ATTCTTTTAG CATAAAGGGA TTTCTTGAGC TGTATCCCAG TAAAAGGGGC GATTTTAGCC AGACTACAAC TCTCTGATTT CGCATACCAG AAGATTGTAT GTGCCCCTAG CCTTCTCTTC ATTGCATTTG TGGCAGAAGA ACTGGCAAAT GCTGTTTTGA TAACCCTTAG CTTGCCAGGG GTGCCTGGGA CCCATGTGTT AGGGGGTGTT ATTAGTGTAC TACAAAAAGA AATCTGGCAC AAAGCCAAAA TAAGTACTAC CAACATAAAT ACAATTTCTA AAGTTGAAAA AAAGAAAAAA AAAGAAAATG AATGTAGGCA GGAGGGCATA CTCACAGTTC ACCCCAGTCC AAGGGTGCAG GCACACAGTA ACATAACCAT ACATATGAGT TCCTCCAAAA AAATAGGACA GAAAGTGAAA AAAAATATGA NdeI

AATATTTATT AGGACTAACG CATTTCGTGC CTGCTGGGGC ACTTACTCAT GAGCCTATGA GTAAGTGCCC CCACAAGCAC GAAATGCGTT AGGCCTTTGC StuI

CAACAAGTAC TAATAAATAT TTAAATGCTG TCCTATTTTT TGGACTAACT CACATTTATG GTTATGTTAC GGTGTTATTG GTGTGTTTGA TCTAAATAAA GTAGACCTTA GCCTCTGCAT ACTTTTAATG GCATATAAAT GATGTCATTA TTAAATATTA TCCCAACCTC ACAGATTGGT GGTCCCTTCA CTCAATAAGG GTAACTGTAC TGCAAGATGA TCGGCCAAGC CCACGTGTAG CTGTTGGGGG TCCCTGTTCC ACTTTCAAAG CGACCAGACT GCTGTCAGTT ATCTGGGGTG GGATAAATGT CTGATCCGCG AAGCTTACAA TTGACGGACT CTACTGTAAA HindIII

TACTGTCTGG GACACTGTAC TGTACACTAT GAATATAAAA CATATTGGGC CTTGAGCGAA AGAAAAGGAG CCGCTATCGC TGATAGGAAA CTCCTGCGCT GTTGTATCAA CAAGCCATTC GGGACCAGCA GAGGGCGCCA GAGTCCAGCA GCTGCCGCTC CGATGTGTTG CGCTGAAAGA CGCGCCCACC GCCGGTCCAC CACTCAAGAG ACGAGGGAGG TG'TCATCCAA GCAACCAACC CTGGCCACGG GCCACCACAC TACTTAAACA TGAATGACAT CACAGCCGTA TGGAGCCTGG CTAATAAATC CTAAGTAGCC AGAGGGACGA GCTACAGACT GGTTGCGGCG CAGGGTTTAT CCAGGGCAGA GAGGAGCAGC AAAAGCACAT TGCGCAGCTC TCACTCCCCC TTTCCTTCTT CTTCACTCTA TAAGGGCTCC TGCAAATGAA AGAGACCTGC GGGGATTTGC GCGGACAGAT GTAAAGGAGA TCCTGCAACT TTCTCTGGTT GCATCCCTGG GAGTCGCTGC GCGCCGCTGG CTGATTGCGA CTGTTGCTTT CCACAGCTCC CTTCTTCCGC AGTTTCTTCT AGGAGCAGAT CGAGTCTCTG GTTACCATGG TGATCGAGCT GAAAGTGAAG AATATTTAAG AGAGGGGAGG CTGGAGCCAG CAGGCAGACA AAGTGGTGCC ACCAAGGACT GTGCGTAAAG GGTGAGCGCA TTGGAGACAG ACAGGGGCTC TGCTGAACTT CCACTTGACT GCGATGAGAG GGGGGAATCC CCAATTCGCT AGGTGCCCCT GAACCCCCCA GAATTC

EcoRI 
bryos demonstraed that the 2066 bp of noggin genomic fragment was indeed able to direct reporter gene to express in vivo, i.e. the noggin genomic fragment we cloned could respond to in vivo signals. Four injected embryos at late neurula stge were shown in Fig 2, demonstrating the reporter gene was expressed primarily in dorsal territories.
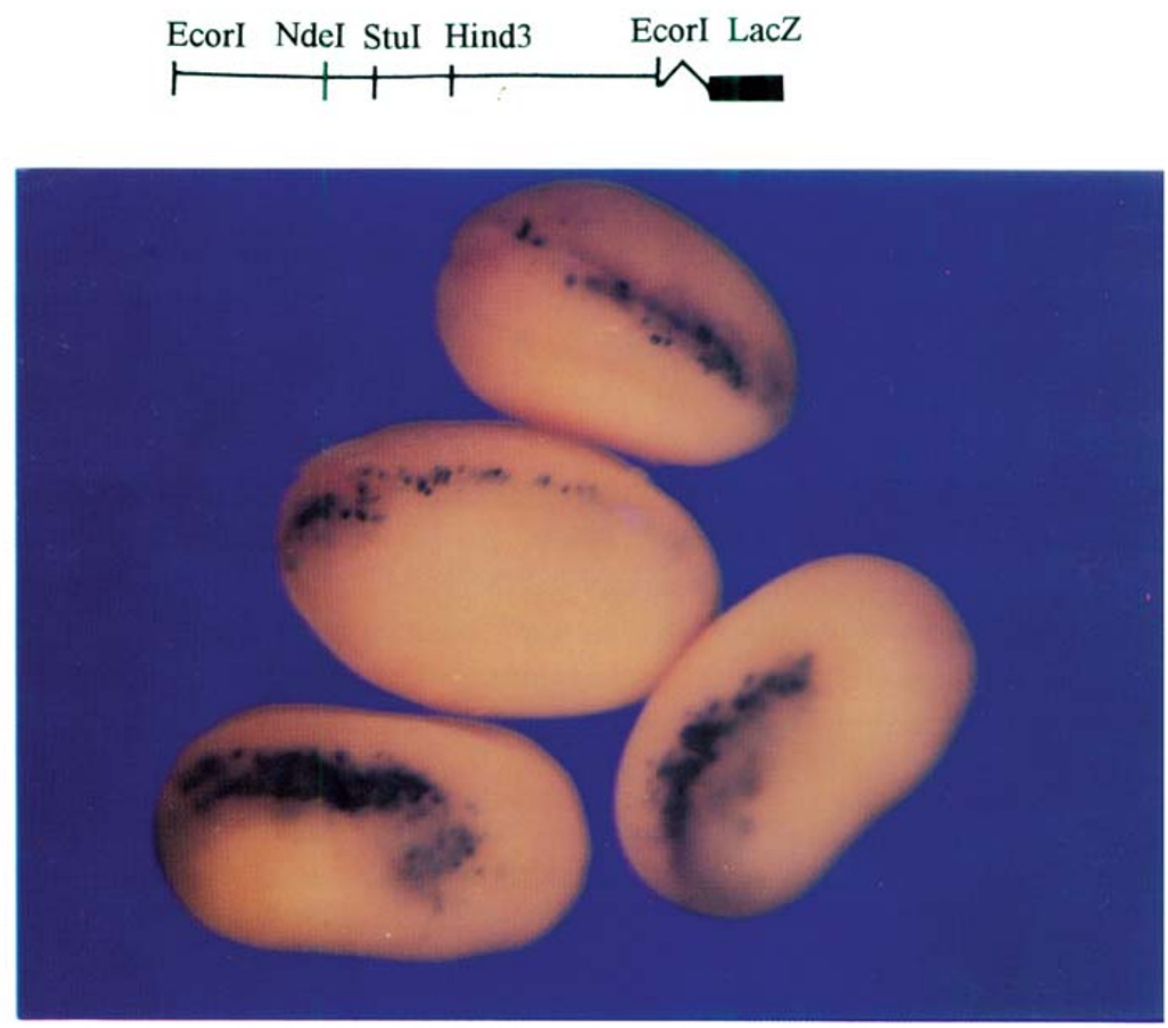

Fig 2.

Showing LacZ reporter construct 2066-LacZ and late neurula stage embryos with $\mathrm{X}$-Gal staining in dorsal territories after injection of 2066-LacZ at 2-cell stage.

\section{$\triangleright$ Fig 1.}

The cloned noggin 5 ' regulatory region and its promoter activity

A. 5' flanking region located in 2-3 kb genomic fractionate (lane 2) with the highest abundance. Lane 1-5 showing the respective PCR results of EcoRI digesteted genomic fragments with 1-2, 2-3, 5-6 kb molecular weight size. Lane 6, EcoRI digested genomic DNA mixture control; Lane 7, genomic control; lane 8, molecular weight standards.

B. 2066 bp 5' upstrem sequence of noggin, including 1550 bp regulatory sequence and 516 bp untranslated region(italics). Two classical TATA boxes are found (1034 bp and $1234 \mathrm{bp}$ ). Restriction endonucleases used for subcloning are indicated. 
5' regulatany region of noggin gene

$1.5 \mathrm{~kb}$ noggin 5' regulatory region is able to response to mesoderm induction signal activin and wnt signals

Since mesoderm inducing factors and doraslizing signals are both required for the formation of the organizer, we tested whether the 5'regulatory region of noggin we cloned could respond to these two kinds signals. Activin, a member of TGF-beta supertamily, was used as mesoderm inducing signal, and ArmdNXTcf-3, a constitutive active transducer of Wnt signaling pathway, was used as dorsalizing signal.

By using the reporter construct 1556-Luc, responsiveness of the 5'regulatory region of noggin to activin or/and ArmdNXTcf-3 was examined in aninimal cap explants. When the reporter construct 1556-Luc was coinjected with activin mRNA, the reporter gene activity was elevated by $70 \%$ compared to that of the injection of 1556-Lac alone (Fig 3, lanes 1 and 2); when it was coninjected with ArmdNXTcf-3 mRNA, the reporter gene activity was 1.5-fold increased (Fig 3, lanes 1 and 3). As control, the promoterless reporter construct pGL3-basic was not responsible to activin and ArmdNXTcf-3 (Fig 3, lanes 4 and 5).

Based on these data, we concluded that in this 1556 bp noggin regulatory region, there did exist relevant cis-elements which enabled noggin to respond to mesoderm inducing and dorsalizing signals. The characterization of the defined response elements for these signals in the noggin gene regulatory region is in progress.

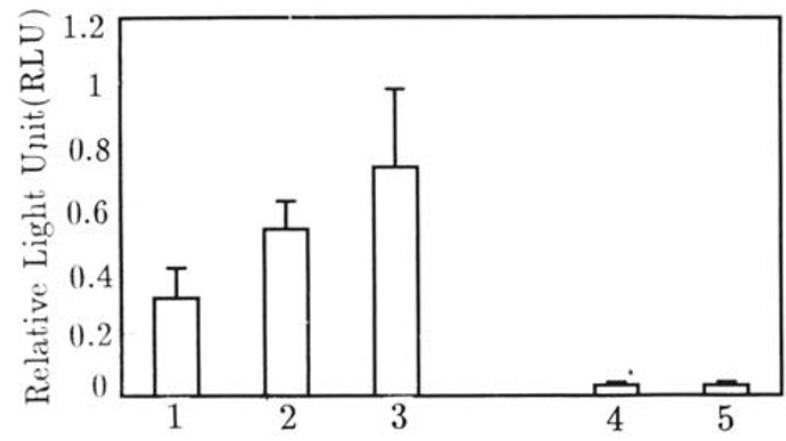

Fig 3.

$1556 \mathrm{bp} 5$ ' regulatory region of noggin is able to response to mesoderm induction signal activin and dorsal determinating signal ArmdNTcf-3.The experiments were repeated three times with repeatable results. Lane 1, reporter activity of 1556-Luc; Lane 2, reporter activity of 1556-Luc coinjected with activin mRNA; Lane 3, reporter activity of 1556-Luc coinjected with ArmdNXTcf3 mRNA; Lane 4, pGL3-basic control; Lane 5, pGL3-basic coinjected with activin and ArmdNXTef-3 mRNAs. 
The data we reported here, together with the findings in the regulatory region of another organizer specific gene goosecoid[8], evidently supported the hypothesis that both mesoderm-inducing and doraslizing signals are involved in the process of organizer formation.

A

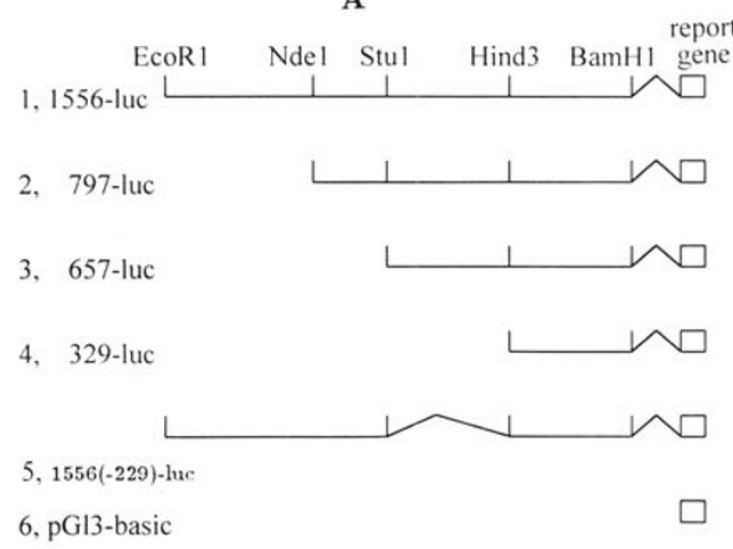

B

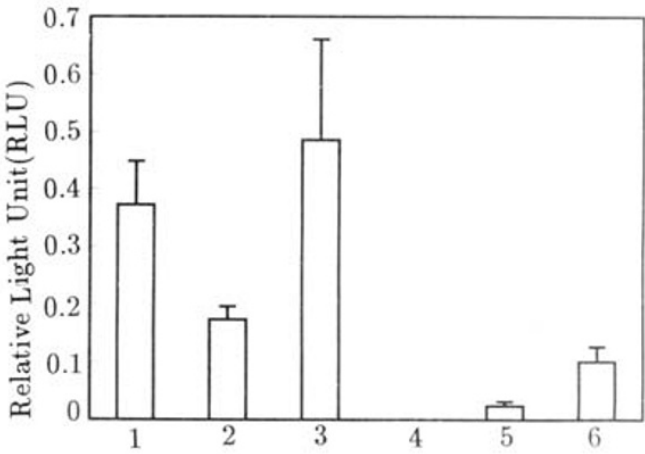

Fig 4.

Dual-luciferase assay to identify the basal promoter of noggin 5 ' regulatory region. The serial deletion reporter constructs were shown in the left part, and the respective ouput of relative luciferase activity was shown in the right part. The experiments were independently repeated more than three times with repeatable results. Only results of one experiment is shown here.

\section{Deletion analysis of noggin promoter region}

The data shown above indicated that the cloned 5' flanking sequence of noggin gene contained promoter activity to regulate the reporter gene expression in vivo and to respond to inducing signals. In order to identify the basal promoter activity of the cloned sequence, we performed the serial deletion analyses by dual-luciferase reporter gene assay (Fig 4). 1556-Luc and its four deletion reporters: 797-Luc, 657-Luc, 329Luc and 1556 (-229)-Luc were injected in dorsal marginal zone at 4-cell stage. The luciferase activities was measured at mid-gastrula stage, when the expression of endogenous noggin reached the peak. The luciferase activities were normalized to internal control pRL-SV40. Consistent with the result of beta-galactosidase reporter gene assay, the promoter activity of 1556 bp sequence was significant (Fig 4). When the sequence distal to the HindIII site was deleted, the remaining $329 \mathrm{bp}$ sequence (329-Luc) showed no promoter activity at all. To further confirm that the $229 \mathrm{bp}$ sequence from HindIII to StuI site played a pivotal role for the promoter activity, we also examined the reporter activity of construct 1550(- 
5' regulatany region of noggin gene

229)-Luc, in which the 229 bp region from HindIII site to StuI site was deleted. No significant difference was found between the reporter activities of 1550(-229)-Luc and 329-Luc. Thus, we concluded that the promoter activity of the 5'regulatory sequence of noggin was located in a 229bp region from HindIII site to StuI site. We also noticed the differences of reporter gene activities between 1556-Luc, 797-Luc and 657-Luc. The relative activities of 1556-Luc and 657-Luc are 2-fold or more higher than that of 797Luc. These data strongly imply that there might be some silencer(s) or enhancer(s) locating in the sequence outside the promoter region.

\section{ACKNOWLDGEMENT}

We are grateful to R.M. Harland for the psp64-activin bb and J Roose for pCDNA1.1ArmdNXTcf-1 constructs. We also thank Mr. Huang Weigong for animal rearing. Ding XY was surported by National Foundation of Natural Science (39770356) and Fundation from Bureau of Basic Research, Chinese Academy of Sciences.

\section{REFERENCES}

[1] Spemman $\mathrm{H}$ and Mangold H. Ueber induktion von embryonalanlagen durch implantation artfremder organisatoren. Welhelm Roux's Arch. Dev Biol 1924; 100:599-638.

[2] Heasman J. Pattening the Xenopus blastula. Development 1997; 124:4179-91.

[3] Smith JC. Mesoderm-inducing factors in early vertebrate development. EMBO J 1993; 12:4463-

[4] Amaya E, Musci TJ and Kirschner MW. Expression of a dominant negetive mutant of the FGF receptor disrupts mesoderm formation in Xenopus embryos. Cell 1991; 66:257-70.

[5] Smith WC and Harland RM Expression cloning of noggin, a new dorsaling factor localized to the Spemman organizer in Xenopus embryos. Cell 1992; 70:828-40.

[6] Ding XY, Hausen P, Steinbeisser H. Pre-MBT patterning of early gene regulation in Xenopus: the role of the cortical rotation and mesoderm induction. Mech Dev 1998; 70:15-25.

[7] Nieuwkoop PD, Faber J. A normal table of Xenopus laevis (Daudin). North Holland Publishing Co., Amsterdam, The Netherlands.

[8] Watabe T, Kim S, Candia A, Rothbaecher U, Hashimoto C, Inoue K and Cho KWY. Molecular mechnisms of Spemann's organizer formation: conserved growth factor synergy between Xenopus and mouse. Genes Dev 1995; 9:3038-50. 